\title{
Light scattering determination of magnetic moments of magnetotactic bacteria (invited)
}

\author{
Charles Rosenblatt, F. Flavio Torres de Araujo, ${ }^{*}$ and Richard B. Frankel \\ Francis Bitter National Magnet Laboratory, Massachusetts Institute of Technology, Cambridge, \\ Massachusetts 02139
}

Light scattering is used to determine the average lengths and magnetic moments of magnetotactic bacteria in
culture. The results are consistent with estimates made from electron

PACS numbers: $75.90 .+\mathrm{w}, 87.10 .+\mathrm{e}, 78.20 . \mathrm{Ls}$

\section{INTRODUCTION}

Magnetotactic bacteria from aquatic sediments orient and swim along magnetic field lines. Each cell contains single magnetic domain $\mathrm{Fe}_{3} \mathrm{O}_{4}$ particles that impart a magnetic dipole moment to the cell, parallel to the axis of motility.2,3 The cells migrate in the field direction (North-seeking) or opposite to the field direction (South-seeking) depending on the orientation of the moment in the cell. The vertical component of the geomagnetic field selects the predominant cell polarity in natural environments. 4,5

In Aquaspirillum magnetotacticum, a freshwater spirillum grown in pure culture 6 the $\mathrm{Fe}_{3} \mathrm{O}_{4}$ particles are typically cuboidal of $400-500 \AA$ dimension. 7 The average number of particles per bacterium can vary from zero to as many as forty depending on the conditions such as the age of the culture, the number of bacteria and the concentration of nutrients and dissolved oxygen The single-magnetic-domain particles are arranged in chains and, because interparticle interactions cause their moments to orient parallel along the chains, the total magnetic moment per bacterium is equal to the sum of the individual particle moments. We report the use of light scattering to determine the average lengths and magnetic moments of bacteria in culture. The results are consistent with estimates of the volume of $\mathrm{Fe}_{3} \mathrm{O}_{4}$ per bacterium from electron micrographs.

\section{EXPERIMENTAL RESULTS}

An electron micrograph of a magnetotactic spirillum is shown in Fig. 1. The electron-opaque particles in the bacterium is the $\mathrm{Fe}_{3} \mathrm{O}_{4}$ that constitutes its biomagnetic compass. The length of the spirillum is 3.4 $\mu \mathrm{m}$. The estimated volume of $\mathrm{Fe}_{3} \mathrm{O}_{4}$ is $9.1 \times 10^{-16} \mathrm{cC}$, with a remanent magnetic moment of $4.4 \times 10^{-13} \mathrm{emu}$. Average lengths and magnetic moments were calculated from thirty micrographs of bacteria from each of two cultures S1 and S2; values are given in Table 1.

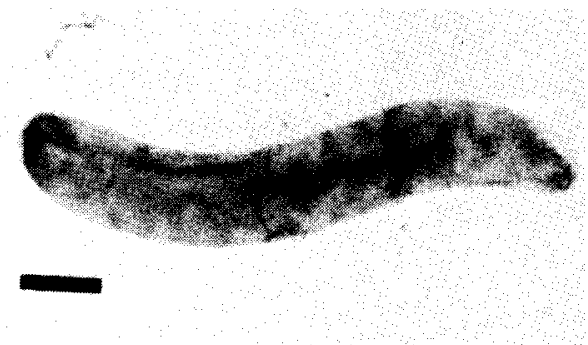

Fig. 1. Electron micrograph of typical bacterium. Dark bar represents $0.5 \mu \mathrm{m}$.
Light scattering was used to determine both the average length 8 and magnetic moment of the bacteria in cultures $\mathrm{S1}$ and S2. Since the volume of $\mathrm{Fe}_{3} \mathrm{O}_{4}$ is only about $1 / 1000$ of the volume of a bacterium, we assume the scattering strength is uniform over the entire bacterium. Thus, modeling the bacteria as optically isotropic cylinders of length $L$ and radius $R$, one can show that the single particle static structure factor is given by

$S_{S P}(Q L, Q R, \bar{\phi})=\left|\frac{\sin \left(\frac{Q L \cos \phi}{2}\right)}{\frac{Q L}{2} \cos \phi} \quad \frac{2 J_{1}(Q R \sin \phi)}{Q R \sin \phi}\right|^{2}$

where $J_{1}$ is the Bessel function of order 1 and $\phi$ is the angle between $\vec{Q}$ and the long axis of the bacterium. $\vec{Q}$ is the momentum transfer of light $\left(\vec{Q}=\vec{k}_{j}-\vec{K}_{f}\right)$. For small diameter bacteria, $Q R \ll 1$ and the term

$2 J_{1}(Q R \sin \phi) /(Q R \sin \phi) \cong 1$; thus the corrections caused by the spiral nature of the bacterium are restricted to a small rescaling of $L$. The assumption of orientational optical isotropy is valid because it was determined that the birefringence of a fully aligned sample in vitro is about $10^{-7}$, and thus should cause only insignificant depolarization effects.

If a magnetic field $\vec{H}$ is applied parallel to $\vec{Q}$, bacteria of magnetic moment $\vec{\mu}$ orient parallel to both $\vec{Q}$ and $\vec{H}$ with an angular distribution function $P(\phi, \alpha)$ given by

$$
P(\phi, \alpha)=\frac{\alpha e^{\alpha \cos \phi} \sin \phi}{2 \sinh \alpha}
$$

where $\alpha=\mu H / k_{B} T, k_{B}$ is Boltzmann's constant, $T$ is temperature, and $\phi$ is defined as before. The total static structure factor $S(Q L, Q R, \alpha)$ is therefore the single particle structure factor, weighted by the angular distribution function, integrated over all angles:

$$
S(Q L, Q R, \alpha)=N \int P(\phi, \alpha) S_{S P}(Q L, Q R, \phi) d \phi
$$

where $N$ is the number of scatterers and $\overrightarrow{0}$ is parallel to $\vec{H}$. The scattered light intensity is thus proportional to $\mathrm{S}(\mathrm{QL}, \mathrm{OR}, \alpha)$.

Three Helmholtz coil pairs oriented at right angles to each other were used to cancel the ambient magnetic field to less than 0.01 Oe at the sample position. A fourth Helmholtz coil pair produced magnetic fields from 0 to 40 0e parallel to $\vec{Q}$ at the sample.

To perform the experiment, the wavevector $Q$ was chosen to be $9898 \mathrm{~cm}^{-1}$, with an acceptance width $\delta Q$ of $500 \mathrm{~cm}^{-1}$ (Fig. 2). The average scattering intensities, measured with a phototube in conjunction with a low pass filter (time constant $t=13.5 \mathrm{sec}$ ), were cor- 


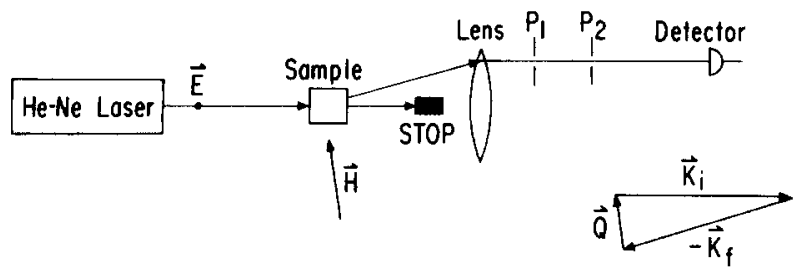

Fig. 2. Schematic representation of scattering experiment (top view). Sample cuvette is $1 \mathrm{~cm}$ in length. The lens has a focal length $f=320 \mathrm{~mm}$ and is placed $320 \mathrm{~mm}$ from the sample. Pinholes $P_{1}$ and $P_{2}$ specify $\vec{Q}$ and are of diameter $d=1.58 \mathrm{~mm}$, spaced $75 \mathrm{~mm}$ apart.

rected for background by first measuring the scattering of water in a cuvette. The water was then removed and the sample was added. The scattering intensities from the bacteria, corrected for background and proportional to $S(Q L, Q R, \alpha)$, were measured in fields $H$ ranging from zero up to $400 \mathrm{e}$. Since $Q \simeq 10^{4} \mathrm{~cm}^{-1}$, $S(Q L, Q R, \alpha)$ is virtualiy independent of $R$, i.e., $S(Q L, Q R, \alpha)=S(Q L, \alpha) . S(Q L, \alpha)$ as calculated from Eq. (3), is shown in Fig. 3 for various values of $\alpha$. As can

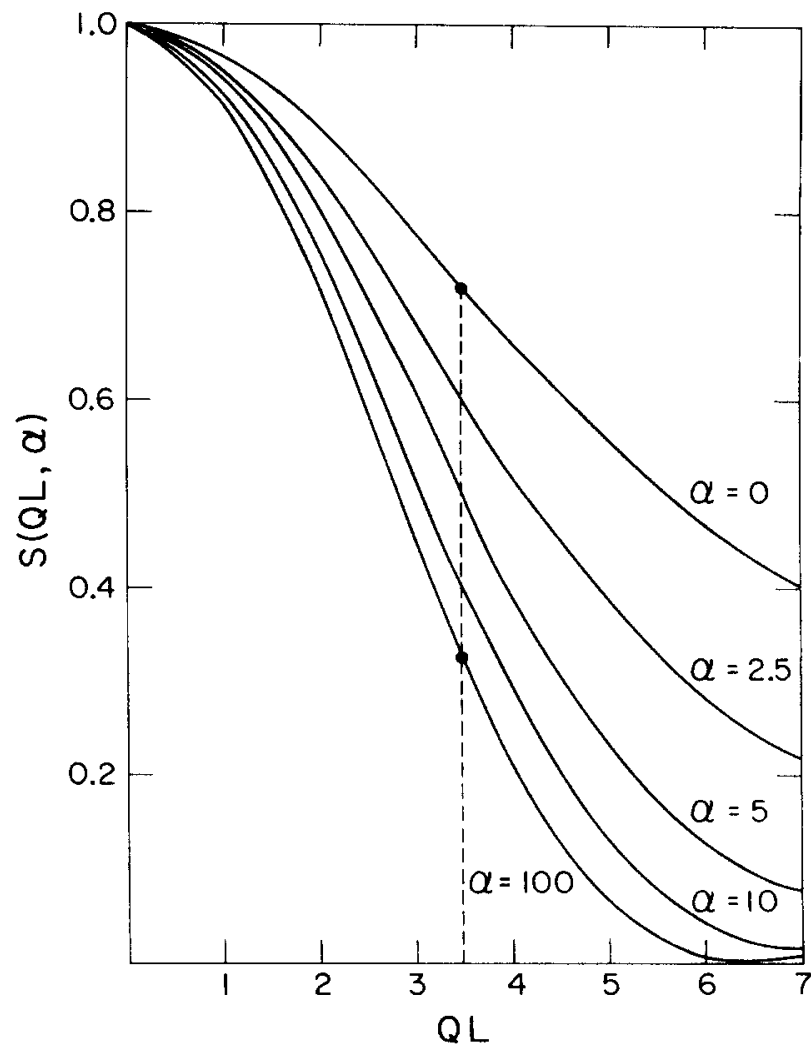

Fig. 3. Structure factor $S(Q L, \alpha)$ vs $Q L$ for various values of $\alpha$, where $\alpha=\mu H / k_{B} T$. The quantity $\mathrm{R}(\mathrm{QL}, \alpha) \equiv \mathrm{S}(\mathrm{QL}, \alpha) / \mathrm{S}(\mathrm{QL}, 0)$ for very large $\alpha$ (saturation) was measured to determine QL; dashed line at $\mathrm{QL}=3.48$ represents one measured culture. Since $Q$ was established by the scattering geometry $\left(Q=9898 \mathrm{~cm}^{-1}\right), a$ value $\langle\mathrm{L}\rangle=3.52 \mu \mathrm{m}$ was obtained. be seen in Fig. 3, if the distribution of lengths is symmetric about some average $\langle L\rangle$ in the vicinity of 2.5 to $5.5 \times 10^{-4} \mathrm{~cm}, \mathrm{~S}(\mathrm{QL}, \alpha)$ is independent of both the acceptance width $\delta Q$ and the distribution width $\delta L$ as long as $\delta Q / Q$ and $\delta L /<L>\leqslant 0.5$.

In zero field the alignment is random and in high field $(\alpha>100)$ it is saturated, with all bacteria aligned parallel to $\vec{H}$. $<L>$ was obtained by measuring $S(O L, \alpha)$ in zero field and in a saturation field of 40 $0 \mathrm{e}$, for which it was later determined that $\alpha \simeq 200$. The quantity $\mathrm{R}(\mathrm{QL}, \alpha) \equiv \mathrm{S}(\mathrm{QL}, \alpha) / \mathrm{S}(\mathrm{QL}, 0)$, which rapidly approaches a limiting value for $\alpha>100$, is a strong function of QL, as can be seen in Fig. 3 by comparing the curves for $\alpha=0$ and $\alpha=100$. Thus a measurement of the intensity ratio $\mathrm{R}(\mathrm{QL}, \alpha)$ for $\alpha>100$ accurately determines $Q L$, and since $Q$ is known from the scattering geometry, $\langle L>$ is determined. In Fig. 3 we show the measured value of $O L$ for culture $S 2$. Values of $\langle L\rangle$ for $\mathrm{S} 1$ and $\mathrm{S} 2$ are given in Table I.

Having determined $\mathrm{OL}$, and therefore $\langle L\rangle$, using the saturation field, $\langle\mu\rangle$ is obtained by measuring $R(Q L, \alpha)$ -t many different fields over the range zero to $40 \mathrm{Oe}$. $n$ Fig. 4, $\mathrm{R}(\mathrm{QL}=3.48, \alpha)$, corresponding to the dashed ine in Fig. 3, is shown. For a given field $H$, the

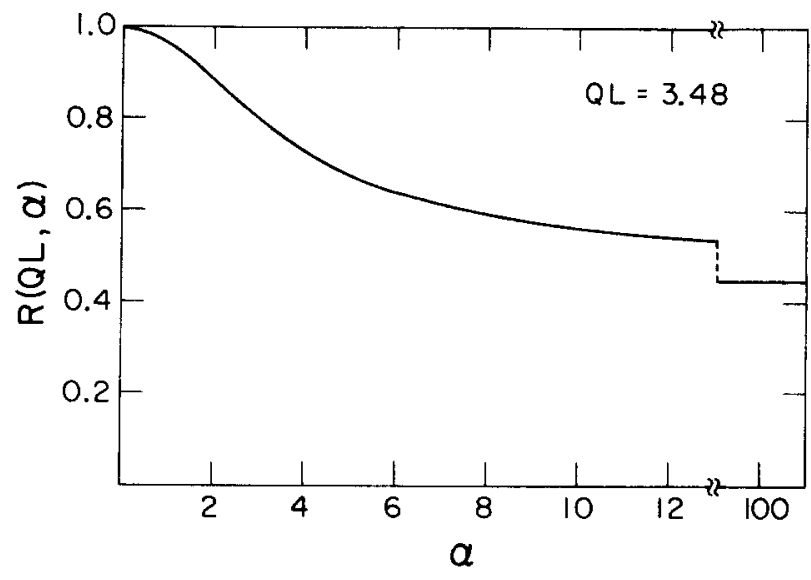

Fig. 4. Theoretical plot of $R(Q L, \alpha)$ vs a for a particular value of $O L(Q L=3.48)$ where $R(Q L, \alpha) \equiv$ $S(Q L, \alpha) / S(Q L, O)$. For a given applied field, $\mathrm{R}(\mathrm{QL}, \alpha)$ was measured, thus establishing $\alpha$. $\langle\mu\rangle=a k_{B} T / H$ was obtained.

value of $\alpha$ corresponding to the measured value of $\mathrm{R}(\mathrm{QL}, \alpha)$ is determined from Fig. 4. Since $\alpha=\mu \mathrm{H} / \mathrm{k}_{\mathrm{B}} T$, and $H$ and $T$ are known, $\langle\mu\rangle$ is obtained. This measurement was performed for approximately ten values of $H$ for each culture and results were averaged to obtain $\langle\mu\rangle$. Values of $\langle\mu\rangle$ are listed in Table I. We note in passing, however, that since $R(O L, \alpha)$ is a strong function of $\alpha$, the distribution width $\delta \mu /<\mu>$ must be less than 0.2 to obtain an accurate measurement of $\langle\mu\rangle$. In addition to the light scattering measurement, bifrefringence technique, similar to that of Scholten, 9 was used to measure $\langle\mu\rangle$. Here the bacteria are assumed to possess an optical polarizability anisotropy $\Delta p$, so that the sample birefringence is

$$
\left.\Delta n=N \Delta p<3 / 2 \cos ^{2} \phi-1 / 2\right\rangle
$$

where $N$ is the number of bacteria and $\phi$ is the angle between $\mathrm{H}$ and the long axis of the bacterium. This technique is insensitive to $L$ and preliminary results for $\langle\mu\rangle$ are consistent with those of the light scattering method. Details will be published elsewhere. 
TABLE I. Parameters of Magnetotactic Bacteria Determined by Light Scattering (L.S.) and Electron Microscopy (E.M.)

\begin{tabular}{llll}
\hline & \multicolumn{1}{c}{ Sample 1 } & Sample 2 \\
\hline $\begin{array}{l}\text { Average Length } \\
\text { (Microns) }\end{array}$ & L.S. & 2.93 & 3.52 \\
& E.M. & $3.2 \pm 0.6$ & $3.4 \pm 0.8$ \\
$\begin{array}{l}\text { Average Magnetic } \\
\text { Moment (emu) }\end{array}$ & L.S. & $(2.2 \pm 0.2) \times 10^{-13}$ & $(4.3 \pm 0.5) \times 10^{-13}$ \\
& E.M. & $2.7 \pm 0.5) \times 10^{-13}$ & $(5.0 \pm 0.8) \times 10^{-13}$ \\
\hline
\end{tabular}

\section{CONCLUSIONS}

Considering the uncertainties in estimating the magnetic moments from the electron micrographs, the agreement of the moments obtained by light scattering with those estimates is quite good. Especially important is the fact that both methods give the same ratio for the average moments in the two samples. Thus static light scattering is a reliable method for rapidly determining the average lengths and magnetic moments of magnetotactic bacteria in culture, and can be used to assay these physical characteristics for bacteria grown under differing culture conditions.

\section{ACKNOWLEDGEMENTS}

We thank Or. R.P. Blakemore for providing culture samples and E. Rhinehart for electron microscopy. The MIT Whitaker College Laboratory of Microscopy provided electron microscope facilities. The Francis Bitter National Magnet Laboratory is supported by the National Science Foundation. This work was partially supported by the Office of Naval Research. F.F.T.A. was supported by the Conselho Nacional de Desenvolvimento Cientifico e Technologico of Brazil.

\section{REFERENCES}

* Permanent Address: Department de Fisica, Universidade Federal do Ceara, Caixa Postal, 1262, 60000 Fortaleza, Ceara, Brasil.

1. R.P. Blakemore, Science 190, 377 (1973).

2. R.B. Frankel, R.P.Blakemore and R.S. Wolfe, Science 203, 1355 (1979).

3. R.B. Frankel and R.P.Blakemore, J. Magn. and Mag. Mater. 15-18, 1562 (1980)

4. R.P. Blakemore, R.B. Franke1, and A.J. Kalmijn, Nature 286, 384 (1980).

5. R.B. Frankel, R.P. Blakemore, F.F. Torres de Araujo, B.M.S. Esquive 1 and J. Danon, Science 212, 1269 (1981).

6. R.P. Blakemore, D. Maratea and R.S. Wolfe, J. Bacteriol. 140, 720 (1979).

7. D.L. Balkwi IT, D. Maratea, and R.P. Blakemore, J. Bacterio1. 141, 1399 (1980).

8. B. Berne and R. Pecora, Dynamic Light Scattering John Wiley, N.Y. , 1976).

9. P.C. Shoiten, IEEE Trans. Magn. MAG-11, 1400 (1975). 